\title{
Cuidados de enfermagem à vítima de acidente vascular cerebral (AVC): Revisão integrativa
}

\author{
Nursing care to the victim of stroke: Narrative integrative
}

Cuidado de enfermería a la víctima de accidente cerebrovascular: Revisión integradora

Dilson Nobre da Silva ${ }^{1-2 \star}$, Mislene Ferreira Xavier de Melo1 ${ }^{1}$ Ériko Marvão Monteiro Duarte ${ }^{1-3}$, Ana Kleiber Pessoa Borges ${ }^{1-4}$.

\section{RESUMO}

Objetivo: Identificar na literatura quais os cuidados de enfermagem no atendimento da vítima sequelado de Acidente Vascular Cerebral. Métodos: Trata-se de uma revisão literária para identificação de diagnósticos e assistências de enfermagem adequados para pacientes acometidos por AVC. A busca dos materiais ocorreu em bases de dados indexados, como PubMed, Scielo e Google Acadêmico, sendo utilizado os descritores: Acidente Vascular Cerebral, Diagnóstico de Enfermagem, Assistência de Enfermagem e Cuidados. Resultados: A amostra final foi constituída por 13 publicações, publicadas entre 2012 e 2019. Considerações Finais: Por meio deste estudo ficou evidente a importância dos cuidados prestados pelo enfermeiro e sua equipe na assistência ao paciente vítima de AVC. Estudos sobre diagnósticos de enfermagem são necessários para que haja melhor conhecimento científico na enfermagem, fundamentando melhor as intervenções de enfermagem voltadas para o controle de resultados satisfatórios, norteando planos de cuidado baseado em evidencias, possibilitando uma promoção de bem-estar e qualidade de vida ao paciente com AVC. Intervenções na prevenção, tratamento e reabilitação são eficazes e condizentes tendo em vista a necessidade da comunidade e do indivíduo, as mesmas devem ser individualizadas, sistematizadas e de qualidade.

Palavras-Chave: Acidente Vascular Cerebral, Diagnóstico de Enfermagem, Assistência de Enfermagem, Cuidados.

\begin{abstract}
Objective: To identify in the literature the nursing care in the care of the sequential victim of Cerebral Vascular Accident. Methods: This is a literary review to identify diagnoses and nursing assistence suitable for patients affected by stroke. The search of the materials took place in indexed detabases, such as PubMed, Scielo and Google Academic, using the descriptors: Cerebral Vascular Accident, Diagnosis of Nursing, Nursing Assistance and Care. Results: The final sample consisted of 13 publications published between 2012 and 2019. Final Considerations: Through this study, it was evident the importance of the care provided by the nurse and his team in the care of the stroke patient. Studies on nursing diagnoses are necessary for better scientific knowledge in nursing, better basing nursing interventions aimed at controlling satisfactory results, guiding evidence-based care plans, enabling a promotion of well-being and quality of life for patients with stroke.
\end{abstract}

Key words: Cerebral Vascular Accident, Diagnosis of Nursing, Nursing Assistance, Care.

\footnotetext{
1'Universidade Federal do Tocantins (UFT), Palmas -Tocantins. *E-mail: dilsonamado@hotmail.com

${ }^{2}$ Hospital Geral de Palmas Dr. Francisco Ayres e Serviço de Atendimento Móvel de Urgência e Emergência (SAMU).

Palmas -Tocantins.

${ }^{3}$ Sistema único de Saúde (SUS). Palmas - Tocantins.

${ }^{4}$ Universidade Estadual Paulista Júlio de Mesquita Filho (UNESP) Rio Claro, SP.
}

SUBMETIDO EM: 10/2019

ACEITO EM: 11/2019

PUBLICADO EM: 11/2019 


\section{RESUMEN}

Objetivo: Identificar en la literatura cuáles son los cuidados de enfermaría en la atención de la víctima secuelada de Accidente Vascular Cerebral. Métodos: Se trata de una revisión literaria para identificar diagnósticos y asistencias de enfermería adecuados para pacientes afectados por AVC. La búsqueda de los materiales ocurrió en bases de datos indexadas, como PubMed, Scielo y Google Académico, siendo utilizado los descriptores: Accidente Vascular Cerebral, Diagnóstico de Enfermería, Asistencia de Enfermería y Cuidado. Resultados: La muestra final consta de 13 publicaciones publicadas entre 2012 y 2019. Consideraciones Finales: A través de este estudio se puso de manifiesto la importancia de los cuidados prestados por el enfermero y su personal en la asistencia al paciente víctima de la apoplejía. Los estudios de diagnóstico de enfermería son necesarios para que haya mejor conocimiento científico en la enfermería, fundamentando mejor las intervenciones de enfermería dirigidas al control de resultados satistactorios, guiando planes de salud baseados en evidencias, posibilitando una promoción del bienestar del paciente con derrame.

Palabras clave: Accidente Cerebral, Diagnóstico de Enfermería, Asistencia de Enfermería, Cuidado.

\section{INTRODUÇÃO}

A partir do investimento na prevenção de doenças infectocontagiosas imunopreveníveis, o perfil epidemiológico brasileiro vem mudando para doenças crônico-degenerativas, como: doenças cerebrovasculares, cardiocirculatórias, endócrinas, carcinogênicas e autoimunes, dentre essas doenças, 0 Acidente Vascular Cerebral (AVC) vem se destacando, já representando a 3ำ principal causa de morte em países industrializados, como o Brasil, e a principal causa de incapacidade entre adultos e idosos (ALMEIDA SEM, 2012).

O AVC é uma doença silenciosa, com grande impacto na população brasileira devido sua grave taxa de morbidade entre o grupo de doenças vasculares, sendo a principal causa de incapacidade e invalidez entre adultos e idosos, chegando a ser fatal em $40 \%$ a $50 \%$ das vítimas após seis meses, onde a maioria dos sobreviventes mostram déficits neurológicos e deficiência residual significativos, tornando o AVC a principal causa da deficiência funcional mundial. O Ministério da Saúde afirma que o AVC é a primeira causa de morte entre as doenças cardiovasculares no Brasil, atingindo principalmente as mulheres (OLIVEIRA DS, 2013).

Nos países em desenvolvimento a incidência das doenças crônicas que não são transmissíveis vem tendo um rápido crescimento quando se comparado com países desenvolvidos, sendo que na atualidade, cerca de dois terços dos Acidentes Vasculares Cerebrais (AVC) ocorrem nesses países. A Hipertensão Arterial Sistêmica (HAS) é a principal causa que oferece risco para o desenvolvimento dessa patologia (LIMA AGT e PETRIBÚ K, 2016; CARVALHO MRS, et al., 2019).

Os AVC podem ocorrer tanto por obstrução de um vaso sanguíneo no cérebro (Derrame Isquêmico), ocorrendo em cerca de $80 \%$ dos casos, como também por hemorragias cerebrais (Derrame hemorrágico), compreendendo os $20 \%$ dos casos restantes (AMORIM DM, 2012).

O Acidente Vascular Cerebral (AVC) está dentro do grupo das principais causas de morte mundial, variando conforme o desenvolvimento socioeconômico do país, acometendo principalmente países subdesenvolvidos ou em desenvolvimento, chegando a atingir um terço da parcela economicamente ativa nesses países. Este cenário se torna preocupante, pois a Organização Mundial de Saúde - OMS estima um aumento de $300 \%$ na população idosa nos países em desenvolvimento até 2025 , quando o Brasil será o sexto país em população idosa mundial (BENSENOR IM, et al., 2015).

Na América Latina, o AVC atinge 150 pessoas a cada 100.000 habitantes, sendo letal em quase metade dos casos. O Ministério da Saúde relata que o AVC é uma das principais causas de óbito em adultos, responsável por $10 \%$ das internações hospitalares públicas, sendo fatal até 12 meses depois em até $40 \%$ dos sobreviventes, pois estes necessitam de reabilitação para as sequelas consequentes do AVC, posto que $70 \%$ 
não retornam ao trabalho e $30 \%$ necessita de auxílio para caminhar, diminuindo severamente a qualidade de vida, entretanto, os últimos avanços na prevenção, atendimento pré-hospitalar e hospitalar, como também no tratamento das sequelas do AVC, trouxeram excepcional melhoria no prognóstico desses pacientes (DE SÁ BP, et al., 2014).

É notório o impacto social, econômico e previdenciário do AVC, porque é um problema de saúde pública que atinge preferencialmente a população adulta e principalmente a idosa, ainda mais aliado a informação de que o Brasil será o sexto país em população idosa no mundo (DALPIAN APC, et al., 2013).

Todo esse cenário exige políticas públicas voltadas para intervir nessa problemática, capacitando profissionais de saúde para essa nova realidade, capazes de atuar em todos os níveis de prevenção, tanto no combate e prevenção do AVC, como também na atenção imediata do atendimento pré-hospitalar e hospitalar, mas também nos cuidados para recuperação das condições de saúde do indivíduo, reabilitando-o para novas condições físicas, melhorando sua qualidade de vida. Portanto, é evidente a importância do profissional de enfermagem no atendimento ao paciente com AVC, tanto no atendimento pré-hospitalar, como também no hospitalar, contribuindo sensivelmente para um melhor prognóstico na alta hospitalar, porque potencializa resultados da reabilitação, minimizando o impacto causado pelas alterações da função sensóriomotora deixadas pelo AVC, promovendo melhor independência funcional e a qualidade de vida nesses pacientes (SOUZA MP e OLIVEIRA IRS, 2012).

Fica evidente que a atuação da enfermagem demonstra grande importância na assistência do paciente com AVC, sendo preciso que se discuta a prática do profissional enfermeiro neste tipo especial de atendimento, identificando os diagnósticos de enfermagem e seus respectivos cuidados. Desta forma esta pesquisa justifica-se pela necessidade de buscar na literatura quais os cuidados que a enfermagem deve ter frente a um paciente vítima de AVC.

\section{MÉTODOS}

Trata-se de uma revisão de literatura, com utilização de livros e artigos indexados em banco de dados. A busca dos materiais para esta revisão foi feita em bases de dados do PubMed (National Library of Medicine), Scielo (Scientific Eletronic Library Online) e Google Acadêmico, sendo utilizado os descritores de ciência e saúde: Acidente Vascular Cerebral, Diagnóstico de Enfermagem, Assistência de Enfermagem, Cuidados. Os critérios de inclusão nesta revisão foram artigos publicados entre 2013 e 2019, disponíveis gratuitamente na integra em português nas bases de dados que abordem a temática do estudo.

Foram excluídos da amostra os artigos cuja publicação antecede ao ano de 2013, que não contemplaram a temática do estudo e que estavam duplicados em ambas as bases de dados. Após a busca nas bases de dados utilizando o cruzamento dos descritores com o operador booleano "and" verificou-se que, conforme ano de publicação entre 2012 e 2019, a base de dado que teve mais publicações foi o Google Acadêmico, resultando em 31.240 publicações. As bases de dados Google Acadêmico, PubMed e Scielo tiveram poucas publicações. O Quadro 2 detalha o quantitativo de publicações encontradas nas bases de dados.

Quadro 1 - Quantitativo de publicações encontradas nas bases de dados.

\begin{tabular}{|c|c|}
\hline BASES DE DADOS & № DE PUBLICAÇÕES \\
\hline Google Acadêmico & 31.140 \\
\hline PubMed & 2 \\
\hline Scielo & 6 \\
\hline TOTAL & 31.148 \\
\hline
\end{tabular}

Fonte: Silva DN, Melo MFX, Duarte EMM, Borges AKP, 2019.

Após levantamento do quantitativo das publicações, foi feita uma leitura superficial do resumo das publicações, seguindo os critérios de inclusão e exclusão, e desta forma a seleção das publicações utilizadas na amostra deste trabalho. Foram eliminados por algum dos motivos de critérios de inclusão e exclusão 31.135 publicações, constituindo como amostra final 13 publicações, como mostra o Quadro 2. 
Quadro 2 - Publicações selecionadas conforme tipo de estudo, objetivo, resultados e publicações entre 2012 e 2019.

\begin{tabular}{|c|c|c|c|c|}
\hline AUTOR & ANO & TIPO DE ESTUDO & OBJETIVO & RESULTADOS \\
\hline $\begin{array}{l}\text { Almeida } \\
\text { SRM }\end{array}$ & 2012 & $\begin{array}{l}\text { Estudo descritivo } \\
\text { de abordagem } \\
\text { quantitativa }\end{array}$ & $\begin{array}{l}\text { Verificar a epidemiologia do acidente vascular } \\
\text { cerebral no Brasil. }\end{array}$ & $\begin{array}{l}\text { Foram registradas } 160.621 \text { internações por doenças } \\
\text { cardiovasculares em } 2009 \text {. Observa-se nas publicações que há } \\
\text { maior prevalência do AVC no gênero feminino }(51,8 \%) \text { em um } \\
\text { grupo de } 2407 \text { pacientes. }\end{array}$ \\
\hline Amorim DM & 2012 & $\begin{array}{l}\text { Estudo transversal, } \\
\text { com o formato } \\
\text { caso-controle, de } \\
\text { caráter } \\
\text { observacional, } \\
\text { descritivo e } \\
\text { analítico }\end{array}$ & $\begin{array}{l}\text { Avaliar os fatores de risco, a frequência de } \\
\text { doença de Chagas, as características clínicas e } \\
\text { etiológicas em pacientes jovens com acidente } \\
\text { vascular cerebral isquêmico e compará-los com } \\
\text { a população de faixa etária mais avançada } \\
\text { acompanhados no mesmo ambulatório de } \\
\text { referência. }\end{array}$ & $\begin{array}{l}\text { Foram avaliados } 45 \text { pacientes com diagnostico de } \mathrm{AVCl} \text {, com } \\
\text { idade entre } 28 \text { a } 78 \text { anos. } 22 \text { (48,9\%) eram do gênero masculino, } \\
\text { houve predomínio da cor de pele negra ( } 52,4 \%) \text {, seguida da parda } \\
\text { ( } 31 \% \text { ) e branca ( } 16,7 \%) \text {, não houve pacientes de origem indígena } \\
\text { e asiática. Fatores de riscos vasculares foram vistos em } \\
\text { proporções variáveis na amostra, } 75,6 \% \text { apresentaram } \\
\text { hipertensão; } 66,6 \% \text { dislipidemia; } 50 \% \text { obesidade; } 31,1 \% \text { diabetes } \\
\text { mellitus; } 20 \% \text { doença arterial coronariana e } 20 \% \text { doença de } \\
\text { chagas. Foi encontrado índice elevado de tabagismo (70,5\%) e } \\
\text { sedentarismo (48,8\%). }\end{array}$ \\
\hline $\begin{array}{l}\text { Botelho TS, } \\
\text { et al. }\end{array}$ & 2016 & $\begin{array}{l}\text { Estudo descritivo, } \\
\text { com abordagem } \\
\text { quantitativa de } \\
\text { caráter explicativo }\end{array}$ & $\begin{array}{l}\text { Verificar o perfil epidemiológico dos pacientes } \\
\text { internados devido o AVC no Brasil no ano de } \\
2014 \text {. }\end{array}$ & $\begin{array}{l}\text { Foram registrados no ano de } 201326.436 \text { internações referentes } \\
\text { ao ataque isquêmico transitório (AIT) e } 130.278 \text { internações } \\
\text { referentes ao AVC não especificado em isquêmico ou } \\
\text { hemorrágico. Houve Maior número de internações do gênero } \\
\text { masculino. }\end{array}$ \\
\hline
\end{tabular}

REAS/EJCH | Vol.Sup.36 | e2136 | DOI: https://doi.org/10.25248/reas.e2136.2019 Página 4 de 11 


\begin{tabular}{|c|c|c|c|c|}
\hline $\begin{array}{l}\text { Bensenor } \\
\mathrm{IM} \text {, et al. }\end{array}$ & 2015 & $\begin{array}{l}\text { Estudo descritivo, } \\
\text { analítico, } \\
\text { quantiqualitativo }\end{array}$ & $\begin{array}{l}\text { Utilizar a Pesquisa Nacional de Saúde (PNS) } \\
\text { aplicada em } 2013 \text { para descrever a prevalência } \\
\text { autodeclarada de AVC na população brasileira } \\
\text { (maior de } 18 \text { anos) com representação para } \\
\text { todo o país, incluindo áreas urbanas e rurais, } \\
\text { de acordo com sexo, raça/cor de pele, nível de } \\
\text { educação le ocupação. }\end{array}$ & $\begin{array}{l}\text { Foram estimadas } 2.231 \text { milhões de pessoas com AVC, sendo as } \\
\text { taxas de prevalência de } 1,6 \% \text { para homens e } 1,4 \% \text { para mulheres. } \\
\text { A prevalência aumentou com o envelhecimento sendo de } 0.1 \% \\
\text { nos estratos de } 18 \text { a } 29 \text { anos e atingindo } 7,3 \% \text { nas faixas etárias } \\
\text { acima de } 75 \text { anos. A prevalência foi maior em indivíduos com } \\
\text { menor escolaridade diminuindo à medida que a escolaridade } \\
\text { aumentava. A prevalência não mudou de acordo com a raça/cor } \\
\text { da pele. A prevalência foi maior nas áreas urbanas em } \\
\text { comparação às rurais. }\end{array}$ \\
\hline $\begin{array}{l}\text { De Sá BP, et } \\
\text { al. }\end{array}$ & 2014 & $\begin{array}{l}\text { Pesquisa causal, } \\
\text { quantitativa, } \\
\text { descritiva, } \\
\text { transversal de } \\
\text { censo }\end{array}$ & $\begin{array}{l}\text { Caracterizar o perfil sócio demográfico e } \\
\text { epidemiológico de pacientes internados por } \\
\text { AVC em um hospital de médio porte no Vale do } \\
\text { Taquari/RS, identificando as doenças } \\
\text { associadas e manifestações clínicas, } \\
\text { possibilitando uma visão ampliada sobre esta } \\
\text { população, com vistas a fornecer informações } \\
\text { para construção de novas estratégias de ação } \\
\text { na prevenção desta. }\end{array}$ & $\begin{array}{l}\text { Durante o período da pesquisa foram constatadas } 9.057 \\
\text { internações pelo SUS, sendo } 479(5,28 \%) \text { por doenças/ou insultos } \\
\text { cardiovasculares. } 125 \text { internações }(40,71 \%) \text { foram por AVC, sendo } \\
63(50,4 \%) \text { do sexo feminino e } 62(49,6 \%) \text { do sexo masculino. A } \\
\text { idade dos pacientes variou de } 21 \text { a } 93 \text { anos. Foram identificados } 8 \\
\text { casos de Ataque Isquêmico Transitório }(6,4 \%), 98 \text { casos de AVCI } \\
(78,45) 19 \text { casos de AVCH }(15,2 \%) \text {. }\end{array}$ \\
\hline $\begin{array}{l}\text { Dalpian } \\
\text { APC, et al. }\end{array}$ & 2013 & $\begin{array}{l}\text { Estudo analítico, } \\
\text { descritivo, } \\
\text { quantiqualitativo }\end{array}$ & $\begin{array}{l}\text { Avaliar o grau de comprometimento da } \\
\text { percepção corporal em pacientes pós-AVC, } \\
\text { através da Scale for Controversive Pushing } \\
\text { (CSCP). }\end{array}$ & $\begin{array}{l}\text { Fizeram parte da amostra oito pacientes do sexo masculino e cinco } \\
\text { do sexo feminino, com idade variada entre } 36 \text { e } 73 \text { anos. Três } \\
\text { pacientes apresentaram AVCH, oito AVCI (62\%) e dois não } \\
\text { sabiam informar e não dispunham de exames para verificação. }\end{array}$ \\
\hline
\end{tabular}

REAS/EJCH | Vol.Sup.36 | e2136 | DOI: https://doi.org/10.25248/reas.e2136.2019 Página $\mathbf{5}$ de 11 


\begin{tabular}{|c|c|c|c|c|}
\hline $\begin{array}{l}\text { Galvão } \\
\text { MTRLS e } \\
\text { janeiro } \\
\text { JMSV }\end{array}$ & 2013 & $\begin{array}{l}\text { Estudo de revisão } \\
\text { teórica da literatura }\end{array}$ & $\begin{array}{l}\text { Compreender melhor a dimensão do conceito } \\
\text { de autocuidado. }\end{array}$ & $\begin{array}{l}\text { O conceito de autocuidado apresenta várias definições gerais, } \\
\text { com uma vasta gama relacionada com a saúde e com a pessoa. }\end{array}$ \\
\hline \begin{tabular}{l|} 
Lima \\
ACMACC, et \\
al.
\end{tabular} & 2016 & $\begin{array}{l}\text { Estudo de revisão } \\
\text { integrativa } \\
\text { literatura }\end{array}$ & $\begin{array}{l}\text { Verificar os diagnósticos de enfermagem } \\
\text { presentes nos pacientes acometidos por AVC. }\end{array}$ & $\begin{array}{l}\text { Foram selecionados nove artigos, sete foram publicados em } \\
\text { periódicos brasileiros. Foi verificado elevado número de } \\
\text { publicações brasileiras realizadas em Fortaleza-CE. }\end{array}$ \\
\hline Oliveira DS & 2013 & $\begin{array}{l}\text { Estudo } \\
\text { observacional, } \\
\text { transversal, } \\
\text { retrospectivo, } \\
\text { quantiqualitativo }\end{array}$ & $\begin{array}{l}\text { Analisar as principais variáveis epidemiológicas } \\
\text { relacionadas ao AVE, no período de } 2009 \text { a } \\
2012 \text {. }\end{array}$ & $\begin{array}{l}\text { As fichas de avaliação foram previamente selecionadas através do } \\
\text { banco de dados disponibilizado pela CLIFOR (Clínica Escola de } \\
\text { Saúde do Unifor), situada no bairro Água Vermelha da cidade de } \\
\text { Formiga-MG. Foram analisadas } 144 \text { fichas, sendo } 63 \text { ( } 43,75 \%) \text { de } \\
\text { indivíduos com AVE, } 38 \text { (60,31\%) eram do gênero masculino e } 25 \\
\text { (39,69\%) feminino. A idade variou de } 14 \text { a } 84 \text { anos, } 27 \text { (42,85\%) } \\
\text { com AVE isquêmico e } 12(19,05 \%) \text { com AVE hemorrágicos. }\end{array}$ \\
\hline $\begin{array}{l}\text { Oliveira } \\
\text { ARS, et al. }\end{array}$ & 2013 & $\begin{array}{l}\text { Estudo descritivo, } \\
\text { transversal }\end{array}$ & $\begin{array}{l}\text { Conhecer o perfil dos pacientes com AVC em } \\
\text { tratamento domiciliar, quanto à capacidade } \\
\text { funcional, estado mental e análise } \\
\text { antropométrica. }\end{array}$ & $\begin{array}{l}\text { A maioria dos participantes era do sexo feminino (59\%), 85,2\% dos } \\
\text { pacientes estavam acamados; } 78,7 \% \text { tinha dificuldade para falar; } \\
70,5 \% \text { não apresentava nenhuma doença além do AVC; } 57,4 \% \\
\text { não praticava nenhuma atividade física; } 36,1 \% \text { apresentava algum } \\
\text { tipo de alteração na pele e usava curativo; } 32,8 \% \text { tinha dificuldade } \\
\text { para ouvir; } 11,5 \% \text { era traqueostomizado e } 9,8 \% \text { fazia uso de } \\
\text { sondagem vesical. }\end{array}$ \\
\hline
\end{tabular}




\begin{tabular}{|l|l|l|l|l|}
\hline $\begin{array}{l}\text { Oliveira } \\
\text { ALR, et al. }\end{array}$ & 2016 & $\begin{array}{l}\text { Estudo de caso, do } \\
\text { tipo descritivo }\end{array}$ & $\begin{array}{l}\text { Realizar a sistematização da assistência de } \\
\text { enfermagem a um paciente sequelado por } \\
\text { AVC, segundo a teoria do déficit do } \\
\text { autocuidado de orem. }\end{array}$ & $\begin{array}{l}\text { O paciente apresentou os principais diagnósticos de enfermagem: } \\
\text { mobilidade no leito prejudicada, tensão do papel do cuidador, risco } \\
\text { de ulcera por pressão, comunicação verbal prejudicada, terapia } \\
\text { com exercício, controle muscular, apoio de tomada de decisão, } \\
\text { treinamento da memória, escutar ativamente, melhora da } \\
\text { comunicação auditiva, fala, visual, saúde emocional do cuidador e } \\
\text { mobilidade física prejudicada. }\end{array}$ \\
\hline $\begin{array}{l}\text { Silva RC, et } \\
\text { al. }\end{array}$ & 2015 & $\begin{array}{l}\text { Estudo de revisão } \\
\text { bibliográfica }\end{array}$ & $\begin{array}{l}\text { Conhecer o papel do enfermeiro frente à } \\
\text { educação permanente, como é importante que } \\
\text { a prática baseada em evidência seja vinculada } \\
\text { à educação da equipe de enfermagem, e como } \\
\text { o enfermeiro é o responsável por promover e e } \\
\text { sustentar essa realidade. }\end{array}$ & $\begin{array}{l}\text { O estudo possibilitou a compreensão que a educação e a pesquisa } \\
\text { se tornam cada vez mais presentes no cotidiano do profissional de } \\
\text { enfermagem. Aliar a pratica baseada em evidências e a procura } \\
\text { pela melhor ação à educação dos profissionais de enfermagem } \\
\text { será umde passo para tornar ainda melhor a qualidade do } \\
\text { cuidado prestado ao paciente. }\end{array}$ \\
\hline $\begin{array}{l}\text { Souza MP e } \\
\text { Oliveira IRS }\end{array}$ & 2012 & $\begin{array}{l}\text { Estudo bibliográfico } \\
\text { descritivo, } \\
\text { abordagem } \\
\text { qualitativa }\end{array}$ & $\begin{array}{l}\text { Descrever a assistência prestada pela } \\
\text { enfermagem no setor de urgência e e } \\
\text { emergência, em âmbito intra-hospitalar, frente } \\
\text { aos pacientes com AVc. }\end{array}$ & $\begin{array}{l}\text { O papel do enfermeiro é o de coordenar, capacitar sua equipe } \\
\text { através de orientações técnicas e auxiliares, para que possa } \\
\text { prestar um atendimento imediato. }\end{array}$ \\
\hline
\end{tabular}

Fonte: Silva DN, Melo MFX, Duarte EMM, Borges AKP, 2019. 


\section{RESULTADOS E DISCUSSÃO}

Com o controle das doenças infectocontagiosas e o envelhecimento da população, cada vez mais os serviços de saúde terão complicações de casos de doenças crônico-degenerativas, como é a situação do AVC (BRASIL, 2013). Os cuidados prestados à vítima de AVC devem necessariamente seguir o padrão: rede básica de saúde, SAMU (Serviço de Atendimento Móvel de Urgência), unidades hospitalares de emergência e leitos de retaguarda, reabilitação ambulatorial, ambulatório especializado, programas de atenção domiciliar, entre outros aspectos. Para o Ministério da Saúde, a doença é uma urgência médica e, como tal, deve ser priorizada em todos os níveis de atenção (BENSENOR IM et al., 2015). A Linha de Cuidado recomenda a criação de unidades específicas para o atendimento ao AVC nos hospitais - as Unidades de AVC -, que comprovadamente diminuem a mortalidade e a incapacidade, além da inclusão do tratamento trombolítico para os casos de AVC isquêmico agudo, único disponível e capaz de reduzir ou até evitar as sequelas (BRASIL, 2013).

Atendendo às recomendações internacionais, a assistência à pessoa acometida por AVC necessita que toda a rede de atenção à saúde esteja articulada, garantindo todos os níveis da atenção. Neste sentido, o enfermeiro precisa estar capacitado para se inserir em qualquer ponto desta rede, prestando uma assistência de enfermagem de qualidade (BRASIL, 2013).

Muitos são os desafios para os enfermeiros que atuam na atenção básica, mas o monitoramento da saúde da população é um dos mais importantes, posto que é a ação que pode identificar as pessoas com perfil suscetível ao AVC, que necessitará da atenção específica de prevenção (BRASIL, 2013).

$\mathrm{Na}$ Atenção Básica o enfermeiro e sua equipe devem realizar ações individuais e coletivas para toda comunidade, promovendo hábitos saudáveis de vida, para prevenção das doenças cardiovasculares, e especificadamente para aqueles com perfil para AVC (BRASIL, 2013). O enfermeiro deve ter conhecimentos para saber aplicar ações específicas, com grande importância nos primeiros socorros prestados à pessoa acometida por AVC, determinando o prognóstico do paciente dentro do ambiente hospitalar (BRASIL, 2013).

Faz-se necessário o mapeamento dos pacientes de risco, para monitoramento de sinais de alerta para AVC, a fim de garantir o acesso aos serviços de saúde, possibilitar o diagnóstico precoce e o tratamento adequado. Em pacientes com queixas sugestivas de AVC, deve-se realizar o primeiro atendimento, avaliar sinais vitais e glicemia (afastando o risco de hipoglicemia), realizar exame neurológico, para então entrar em contato com a central de regulação de urgência para encaminhamento do usuário com acompanhamento especializado e multiprofissional (BENSENOR IM, et al., 2015).

Deve-se suspeitar de AVC sempre que o paciente apresentar início súbito de déficit focal, com ou sem alteração do nível de consciência, mantendo atenção especial aos sinais de alerta: perda de força ou formigamento de um lado do corpo; dificuldade em falar ou compreender; perda visual em um ou ambos os olhos; tontura, perda de equilíbrio e ou de coordenação e Dor de cabeça súbita, intensa sem causa aparente (PADILHA ARS, 2011).

É recomendado pelo Ministério da Saúde o uso de uma escala pré-hospitalar para avaliação de AVC, que deve ser aplicada para reconhecer os sinais mais frequentes, sendo: avaliação da face, pedindo ao paciente que dê um sorriso e assim observar se há algum desvio da boca; avaliação da força, solicitando que o paciente ele os dois braços e observando se há que não consegue manter elevado devida a perda da força; e observar a fala, solicitando ao paciente que repita alguma frase, como "O céu é azul" e observar se há alteração na fala (BRASIL, 2013).

$\mathrm{Na}$ assistência pré-hospitalar inicial o enfermeiro deve prestar atendimento clínico conforme os seguintes cuidados: verificar os sinais vitais (pressão arterial, pulso, saturação, temperatura axilar); posicionar a cabeceira a 0 grau (exceto se vômitos. Neste caso manter cabeceira a 30 graus); realizar acesso venoso periférico em membro superior não parético; administrar oxigênio por cateter nasal ou máscara, se oximetria 
$<92 \%$; checar glicemia capilar - hipoglicemia pode causar sinais focais e simular um AVC. Se glicose $<70$ $\mathrm{mg} / \mathrm{dl}$ (miligramas por decilitro) administrar glicose hipertônica $50 \% 20 \mathrm{ml}$ (mililitro); aplicar a escala préhospitalar de AVC (BRASIL, 2013).

Deve-se também determinar a hora do início dos sintomas e sinais, que pode ser referida pelo paciente (se este estiver orientado e coerente) ou pelo acompanhante; reduzir níveis pressóricos quando pressão arterial sistólica $\geq 220 \mathrm{mmHg}$ ou pressão arterial diastólica $\geq 120 \mathrm{mmHg}$, ou se outra doença associada exija a redução da pressão arterial, como: dissecção de aorta, infarto agudo do miocárdio, edema pulmonar; administrar grande volume de fluido em caso de hipotensão, utilizando soro fisiológico 0,9\%; utilizar antitérmico se temperatura axilar $>37,5^{\circ} \mathrm{C}$, recomendada Dipirona $1 \mathrm{~g}$ (grama) EV (endovenoso); e priorizar o Resgate de paciente com AVC com ambulância no domicílio ou nas unidades de pronto atendimento ou serviços 24horas (BRASIL, 2013).

Após atendimento inicial cabe ainda ao enfermeiro notificar o hospital de destino; levar o acompanhante (preferencialmente que tenha presenciado o início dos sintomas) para o hospital; seguir protocolo em pacientes não candidatos à terapia trombolítica, enquanto aguardam nas UPA (Unidade de Pronto Atendeimento) 24horas por remoção ao serviço referenciado para AVC; suspender dieta até avaliação da capacidade adequada de deglutição; realizar teste de triagem para disfagia quando não houver possibilidade de transferência do paciente. Caso o teste tenha resultado normal, iniciar medicação oral e dieta pastosa hipossódica, cabeceira em 90 graus, sob supervisão (BRASIL, 2013).

Outras intervenções assistenciais também devem ser realizadas, como, manter hidratação venosa com venóclise de solução fisiológica a 0,9\% EV contínuo para facilitar administração de medicações; administrar antitérmico caso temperatura axilar $\geq 37,5^{\circ} \mathrm{C}$ com Paracetamol 500 miligrama VO (Via Oral) (preferência) ou Dipirona $1 \mathrm{~g} \mathrm{EV}$; administrar AAS (Ácido Acetilssalicílico) 300mg VO em 24h e Captopril 25 mg VO 6/6 horas se PAS (Pressão Arterial Sistólica) $\geq 220 \mathrm{mmHg}$ ou PAD (Pressão Arterial Diastólica) $\geq 120$ $\mathrm{mmHg}$, ou se outra condição clínica exigir (BRASIL, 2013).

Durante o período de internação do paciente na unidade hospitalar, o enfermeiro e sua equipe são responsáveis por prevenir complicações relacionadas ao AVC, desta forma devem aplicar intervenções como promover mobilidade e prevenir deformidades, prevenir a adução e dor do ombro, promover mudança de posições, estabelecer um programa de exercícios, tratar a disfagia, obter o controle intestinal e vesical, melhorar a comunicação e manter a integridade cutânea (OLIVEIRA ALR, et al., 2016).

Algumas medidas podem ser aplicadas para melhores resultados nas intervenções, como por exemplo, por meio do posicionamento correto, usando medidas para avaliar a pressão, auxiliar no controle do bom alinhamento corporal e evitar neuropatias compressivas, especialmente dos nervos olhar e fibular. $O$ uso de um travesseiro quando colocado na axila limita a rotação externa, mantendo o braço afastado do tórax. A posição do paciente deve ser mudada a cada duas horas, para colocar um paciente em uma posição lateral (decúbito lateral), coloca-se um travesseiro entre as pernas antes que o paciente seja virado. (OLIVEIRA ALR, et al., 2016).

O paciente deve ser estimulado a movimentar os membros oito a cinco vezes por dia, de forma a manter a mobilidade articular, recuperar o controle motor, evitar a deterioração adicional do sistema neuromuscular e estimular a circulação. O exercício é valioso na prevenção da estase venosa, a qual pode predispor o paciente à trombose e embolia pulmonar. É importante lembrar o paciente de exercitar o lado não afetado a intervalos durante todo o dia (OLIVEIRA ALR, et al., 2016).

Durante o processo de reabilitação da vitíma de AVC, o enfermeiro é o responsável por: desenvolver um processo interacional e transdisciplinar que favoreça o planejamento de recuperação do paciente; proporcionar o envolvimento e a participação ativa e sistemática do paciente e sua família aos cuidados a serem desempenhados em casa (DIOGO MJD, 2000; GOMES SRG e SENNA M, 2008). 
Além dos cuidados prestados, é de responsabilidade do enfermeiro promover educação em saúde para toda a comunidade, tendo em vista que esta prática promove o autocuidado do indivíduo, uma vez que introduz e/ou reforça comportamentos de proteção e manutenção da saúde, permitindo, deste modo, reflexões para a melhoria da qualidade de vida (TAVARES DMS, et al., 2012).

Diante do papel do enfermeiro na assistência à vitíma de AVC pode-se obter alguns diagnósticos e intervenções de Enfermagem, sendo: ansiedade relacionada à morte, por fatores situacionais evidenciados por medo da perda de capacidades físicas e/ou mentais; risco para constipação relacionado à falta de conscientização para a ingesta hídrica; medo relacionado à perda de controle e aos resultados imprevisíveis secundários à falta de conhecimento da patologia evidenciado por relatos verbais do paciente; hipertensão relacionada à doença; nutrição alterada menor que as necessidades corporais relacionada à dificuldade funcional de se alimentar; risco para integridade da pele prejudicada relacionada à dificuldade de mobilidade e risco de queda; e déficit de conhecimento sobre a natureza de sua doença e tratamento relacionado com não familiaridade com os recursos de informação (LIMA ACMACC, et al., 2016.)

Com o diagnóstico de enfermagem pode-se propor intervenções para melhoramento do bem-estar e recuperação de saúde do paciente. Sendo assim são possíveis intervenções como permitir que a pessoa compartilhe suas percepções sobre a situação; orientar quanto à doença e seus aspectos; solicitar apoio psicológico, se necessário; insistir na importância para uma dieta equilibrada, sugerir caminhada; incentivar ingestão hídrica; oferecer fluidos preferidos; orientar o paciente a tirar possíveis dúvidas e encorajar a expressão dos sentimentos e respostas que reflitam realidade (LIMA ACMACC, et al., 2016).

É valido ainda ensinar técnicas de relaxamento; verificar sinais vitais regularmente, atenção especial com pressão arterial; proporcionar ambiente arejado; orientar quanto à alimentação; estimular a ingestão de alimentos preferidos, tendo atenção a restrições da prescrição médica; recomendar refeições pequenas e com maior frequência; promover restrição hídrica e de sódio na dieta; monitorar o surgimento ou piora da condição de saúde; Observar a diurese diária; instruir o paciente sobre dados relativos à doença; esclarecer acerca do plano terapêutico; fornecer informações sobre o potencial das complicações; contatar a família para obter sua participação; encorajar o paciente a manter-se independente e ncentivar o engajamento em atividades de lazer (LIMA ACMACC, et al., 2016).

\section{CONSIDERAÇÕES FINAIS}

Por meio deste estudo ficou evidente a importância dos cuidados prestados pelo enfermeiro e sua equipe na assistência ao paciente vítima de AVC. Estudos sobre diagnósticos de enfermagem são necessários para que haja melhor conhecimento científico na enfermagem, fundamentando melhor as intervenções de enfermagem voltadas para o controle de resultados satisfatórios, norteando planos de cuidado baseado em evidencias, possibilitando uma promoção de bem-estar e qualidade de vida ao paciente com AVC. Intervenções na prevenção, tratamento e reabilitação são eficazes e condizentes tendo em vista a necessidade da comunidade e do indivíduo, as mesmas devem ser individualizadas, sistematizadas e de qualidade.

\section{REFERÊNCIAS}

1. ALMEIDA SEM. Análise epidemiológica do acidente vascular cerebral no Brasil. Rev. Neurocienc. v.20 n.2, 2012. p. 481-482.

2. AMORIM DM. Características clínicas e fatores de riscos em pacientes jovens com acidente vascular cerebral. Salvador: UFBA, 2012. (Trabalho de Conclusão do Curso de Medicina da Universidade Federal da Bahia).

3. BRASIL. Ministério da Saúde. Secretaria de Atenção à Saúde. Departamento de Ações Programáticas Estratégicas. Diretrizes de atenção à reabilitação da pessoa com acidente vascular cerebral. Brasília: Ministério da Saúde, 2013.

4. BOTELHO TS, et al. Epidemiologia do acidente vascular cerebral no Brasil. Temas em saúde, João Pessoa, 2016. 
5. BENSENOR IM, et al. Prevalência de avc e incapacidade associada no Brasil: pesquisa nacional de saúde. Arq. Neuro-Psiquiatr. v.73 n.9, set. 2015. p.746-750.

6. CASTILHO AP, et al. Manual de metodologia científica. Itumbiara: ILES/ULBRA, 2014.

7. CARVALHO MRS, et al. Cuidados de Enfermagem ao Paciente acometido por Acidente Vascular Cerebral: Revisão Integrativa. Rev. Mult. Psic. V.13, N. 44, p. 198-207, 2019.

8. DE SÁ BP, et al. Perfil de pacientes internados por acidente vascular cerebral em hospital do Vale do Taquari RS. Rev. Neurocienc. v.22 n.3, 2014. p.381-387.

9. DALPIAN APC, et al. Avaliação da percepção corporal em pacientes pós-acidente vascular cerebral. Rev. Neurocienc., v.21 n.3, 2013. P.377-382.

10. DIOGO MJD. O papel da enfermeira na reabilitaação do idoso. Rev Latino-Am Enferm. 2000.

11. GALVÃO MTRLS, JANEIRO JMSV. O autocuidado em enfermagem: autogestão, automonitorização e gestão sintomática como conceitos relacionados. REME Rev. Min. Enferm., Jan. /mar., 2013.

12. GOMES SRG, SENNA M. Assistência de Enfermagem à pessoa com Acidente Vascular Cerebral. 2008.

13. HINKLE JL, CHEEVER KH. Brunner \& Suddarth - Tratado de Enfermagem Médico-Cirúrgica. 13 ed. Rio de Janeiro, Guanabara, 2016.

14. LAKATOS EM, MARCONI MA. Fundamentos de metodologia científica. 8ed. São Paulo: Atlas, 2017.

15. LIBARIO D, TERRA I. Metodologia científica, São Paulo: Rede Internacional de Universidades Laureate, $2015 . Z$

16. LIMA ACMACC; et al. Diagnósticos de enfermagem em pacientes com acidente vascular cerebral: revisão integrativa. Rev. Bras. Enferm. jul./ago., 2016.

17. LIMA AGT, PETRIBÚ K. Acidente vascular encefálico: revisão sistemática sobre qualidade de vida e sobrecarga de cuidadores. Revista Brasileira de Neurologia e Psiquiatria. v. 20, n. 3, p.253-266, Set/dez., 2016.

18. OLIVEIRA DS. Análise do perfil epidemiológico de pacientes com acidente vascular encefálico atendidos na clínica escola de saúde do UNIFOR-MG. Formiga: UNIFOR-MG, 2013. (Trabalho de conclusão de curso apresentado ao curso de Fisioterapia do Centro Universitário de Formiga UNIFOR-MG).

19. OLIVEIRA ARS, et al. Avaliação de pacientes com acidente vascular cerebral acompanhados por programas de assistência domiciliária. Rev EsC Enferm USP. 2013.

20. OLIVEIRA ALR, et al. Assistência de enfermagem a um paciente sequelado por acidente vascular cerebral no domicílio baseado na teoria de Orem. Mostra Interdisciplinar do curso de Enfermagem, 2016.

21. PADILHA ARS. Implantando a linha de cuidado do Acidente Vascular Cerebral- AVC na Rede de Atenção às Urgências. 2011.

22. SOUZA MP, OLIVEIRA IRS. Enfermagem na assistência ao paciente com acidente vascular cerebral em ambiente intra-hospitalar. Três Rios: Faculdade Redentor, 2012. (Monografia do Curso de Especialização em Urgência E Emergência).

23. SILVA RC, et al. O papel do enfermeiro como educador e pesquisador, e a integração entre prática baseada em evidências e educação permanente. Percurso Acadêmico, Belo Horizonte, jul./dez., 2015.

24. SANTOS IE. Manual de métodos e técnicas de pesquisa científica. 11ed. Rio de Janeiro, Impetus, 2015.

25. TAVARES DMS, et al. Qualidade de vida de idosos e participação em atividades Educativas grupais. Acta Paulista de Enfermagem, São Paulo, Jul. 2012. 